\title{
High-Risk Immunization Strategies for Multiethnic Regions
}

\author{
Fuzhong Nian and Tong Liu \\ School of Computer \& Communication, Lanzhou University of Technology, Lanzhou 730050, China \\ Correspondence should be addressed to Fuzhong Nian; gdnfz@lut.cn
}

Received 8 January 2014; Accepted 24 February 2014; Published 7 April 2014

Academic Editor: Fuding Xie

Copyright (C) 2014 F. Nian and T. Liu. This is an open access article distributed under the Creative Commons Attribution License, which permits unrestricted use, distribution, and reproduction in any medium, provided the original work is properly cited.

\begin{abstract}
In the Multiethnic regions, like the west of China, because of the difference of religious beliefs, ethnic customs, and mode of production, the contacts and relationships are also different. The epidemic characteristics of these regions are different from other places. Based on the background, some high-risk immunization strategies for Multiethnic regions are proposed. The epidemic dynamics were analyzed both from theory and simulation experiment. The results indicate that the proposed immunization strategies are effective, and it is also economic and feasible.
\end{abstract}

\section{Introduction}

The problem of epidemic spreading has been a hot topic in past decades. Infectious diseases occur frequently in the $21 s t$ century, for example, SARS in 2003, the bird flu in 2005, H1N1 in 2007, and so forth. All these seriously threaten the health and life of the people. Recently, various epidemic models were investigated [1-6]. In typical epidemic mode, Individuals were divided into several classes. Generally, the basic states include $S$ standing for susceptible state, $I$ standing for infected state, and $R$ standing for recovered state. The typical mode is named by the transform of the states, such as the susceptibleinfected-susceptible (SIS) [7], susceptible-infected-recovered (SIR) [8], and susceptible-infected (SI) [9]. Around these models, various problems have been investigated [3, 10-16].

As for epidemic dynamics, one key problem is whether the epidemic propagation can be effectively controlled by vaccination aiming at part of the population. At present, there are two methods to solve the problem. One is to make infected individuals isolated and treat them; the other is to carry out vaccination of susceptible individuals. Because the isolation and treatment not only often bring many inconveniences but also are very unfavorable to the social economic development, so people generally use the method of vaccination.

Based on mean-field theory, Callway proposed a random immunization in which the nodes to be vaccinated are selected randomly [17]. However, experiments indicate that almost all individuals need to be vaccinated for controlling the epidemic. Obviously, it is impossible in practice. In order to solve the problem, Pastor-Satorras and Vespignani investigated network immunization for SIS model and proposed another scheme-called "targeted immunization schemes" [18], in which the nodes with larger degrees are vaccinated firstly. The efficiency of the targeted immunization schemes is much higher than the former. However, the targeted immunization schemes require all of information of the whole network before being implemented. In response to this issue, Cohen proposed an efficient immunization strategy named "acquaintance immunization" [8]. In this scheme, the "acquaintances" of some nodes which are selected randomly are vaccinated with certain probability. It is evident that the nodes with large degrees have more chance to be vaccinated. However, all above works are done based on the hypothesis that all individuals are uniform. In other words, the traffic capacities of all edges of the networks are equal. But it is not true in fact. For example, at the multiethnic regions, the contact frequency of two individuals who come from the same nationality is bigger than the situation from different nationalities. Nodes in networks are not always from the same nationality. In this paper, the nodes in the network are divided into three classes which represent the individuals coming from three different nationalities. Besides, all these immunizations do consider not only whether an immunization strategy is effective or not, but also whether the immunization strategy is feasible or not. Uniform immunization is a simple 
strategy and can be done in the real life. But it has a clear drawback which needs to vaccinate almost all nodes; the cost of requirement is huge. Obviously, it is not efficient. We need a simple and effective strategy to make sure of vaccinating less people and getting better performance. The most important thing is whether the selected strategy can be adopted in real life.

In consideration of the above problems, we studied highrisk immunization strategies for multiethnic regions which are to vaccinate the susceptible individuals whose neighbors have been infected. And these individuals are called "highrisk individuals" [16]. Is this immunization valid? According to the mean-field theory, we build the standard SIRS model on BA scale-free networks in which all nodes come from different nationalities and investigate the behaviors of density of infected individuals.

The rest of the paper is organized as follows. In the next section, high-risk immunization in different BA scale-free complex networks is investigated, and the standard SIRS model is built. Finally, conclusions are given in the last section.

\section{Immunization in Scale-Free Networks}

A major discovery in the study of complex networks is that many networks including Internet, WWW, and metabolic networks have power law distribution in recent years. In order to explain the appearance of power law distribution, Barabási and Albert proposed a new scale-free network called BA. They use several steps to build BA scale-free network. A small number of $m_{0}$ nodes will be constructed first, and then a new node is added in every step, with $m$ links that are connected to an old node $i$ with probability $\prod\left(k_{i}\right)=k_{i} / \sum_{j} k_{j}$, where $k_{i}$ is the degree of the $i$ th node. When the process cycles enough, we obtain a network composed of $N$ nodes with degree distribution $P(k) \sim k^{-3}$ and average degree $\langle k\rangle=2 m$. In a concrete spreading model, each node in the network represents an individual, and each link is an approach to spread viruses. Each susceptible node is infected with rate $\beta$, if it is connected to one or more infected nodes. Infected nodes are cured with rate $\alpha$. Defining an effective spreading rate $\lambda=\beta / \alpha$, without loss of generality, we set $\alpha=1$. A recovered node loses its immunity with rate $\sigma$. For this class of graphs, it has significant scale-free property; nodes with high degrees play an important role in network; so they can not be neglected. We should relax the homogeneity assumption used for homogeneous networks. We consider the relative density $S_{k}^{i}(t)$ of susceptible nodes, $I_{k}^{i}(t)$ of infected nodes, and $R_{k}^{i}(t)$ of recovered nodes with given degree $k$ in the $i$ th region; that is, the probability that a node with $k$ links is susceptible, infected, or recovered is $S_{k}^{i}(t), I_{k}^{i}(t)$, and $R_{k}^{i}(t)$, respectively. Suppose that there are $n$ different ethnics; thus, the SIRS model can be modified as follows:

$$
\begin{aligned}
& \frac{d S_{k}^{i}(t)}{d t}=-\lambda k\left(1-\frac{\delta \Omega}{\sigma}\right) \Theta(t) \sum_{i=1}^{n} \zeta_{i j} S_{k}^{i}(t)+\sigma R_{k}^{i}(t) \\
& \frac{d I_{k}^{i}(t)}{d t}=\lambda k\left(1-\frac{\delta \Omega}{\sigma}\right) \Theta(t) \sum_{i=1}^{n} \zeta_{i j} S_{k}^{i}(t)-I_{k}^{i}(t)
\end{aligned}
$$

$$
\begin{aligned}
\frac{d R_{k}^{i}(t)}{d t} & =I_{k}^{i}(t)-\sigma R_{k}^{i}(t) \\
\zeta_{i j} & = \begin{cases}1 & (i=j) \\
\tau & (i \neq j) .\end{cases}
\end{aligned}
$$

Here, $\zeta_{i j}$ represents impact coefficient between two different ethnics, $0<\tau<1$. The first term on the rhs of the first equation of (1) considers the average density of newly infected nodes generated by each infected node. This is proportional to the effective infection spreading rate $\lambda$, the number of connections $k$ (degree), the probability that a given link points to a healthy node $S_{k}^{i}(t)$, and the probability $\Theta(t)$ that any given link points to an infected node. The second term represents recovered nodes losing immunity with rate $\sigma$. The last term represents the change from susceptible nodes to recovered nodes by vaccinating. This is proportional to the vaccinating rate $\delta$, the probability that a given link points to a healthy node $S_{k}^{i}(t)$, the probability that a given node is a neighbor of specific node $\Omega$, and the probability $\Theta(t)$ that any given link points to an infected node. The second term on the rhs of the second equation of (1) denotes the infected nodes recovering with unit rate. The explanations of other terms in (1) are similar to the above. The probability that a link points to a node with s links is proportional to $s P(s)$. In other words, a randomly chosen link is more likely to be connected to an infected node with high connectivity, yielding

$$
\Theta(t)=\frac{\sum_{k} k P(k) I_{k}^{i}(t)}{\sum_{s} s P(s)}=\frac{1}{\langle k\rangle} \sum_{k} k P(k) I_{k}^{i}(t) .
$$

Also, $S_{k}^{i}(t), I_{k}^{i}(t)$, and $R_{k}^{i}(t)$ obey the normalization condition for each $k$ :

$$
S_{k}^{i}(t)+I_{k}^{i}(t)+R_{k}^{i}(t)=1 .
$$

Consider the nonepidemic stationary condition:

$$
\begin{aligned}
\frac{d S_{k}^{i}(t)}{d t} & =0 \\
\frac{d I_{k}^{i}(t)}{d t} & =0 \\
\frac{d R_{k}^{i}(t)}{d t} & =0 \\
I(t) & =0 .
\end{aligned}
$$

Then, from the first equation of (1), we obtain

$$
-\lambda k\left(1-\frac{\delta \Omega}{\sigma}\right) \Theta(t) \sum_{i=1}^{n} \zeta_{i j} S_{k}^{i}(t)+\sigma R_{k}^{i}(t)=0 .
$$

By combining the above equation with (3), we obtain

$$
S_{k}^{i}(t)=\frac{\sigma-\sigma I_{k}^{i}(t)}{-\lambda k(1-\delta \Omega / \sigma) \Theta(t)\left(2 \sum_{i}^{n} \zeta_{i j} /\left(n^{2}+n\right)\right)+\sigma} .
$$


From the second equation of (1),

$$
S_{k}^{i}(t)=\frac{I_{k}^{i}(t)}{\lambda k(1-\delta \Omega / \sigma) \Theta(t)} \frac{2 \sum_{i}^{n} \zeta_{i j}}{n^{2}+n} .
$$

Substitute (7) into (6),

$$
I_{k}^{i}(t)=\frac{\sigma \lambda k(1-\delta \Omega / \sigma) \Theta(t)\left(2 \sum_{i}^{n} \zeta_{i j} /\left(n^{2}+n\right)\right)}{(1+\sigma) \lambda k(1-\delta \Omega / \sigma) \Theta(t)\left(2 \sum_{i}^{n} \zeta_{i j} /\left(n^{2}+n\right)\right)+\sigma} .
$$

The solutions $I_{k}^{i}(t)=0$ and $\Theta(t)=0$ always satisfy (8). A nonzero stationary prevalence $\left(I_{k}^{i}(t) \neq 0\right)$ is obtained when the lhs and the rhs of (8) are expressed as function $F(\Theta)$, and interest is in the interval $0<\Theta \leq 1$; this has a nontrivial solution. It is easy to deduce that this requires that the inequality

$$
\left.\frac{d F(\Theta)}{d \Theta}\right|_{\Theta=0} \geq 1
$$

must be fulfilled; that is,

$$
\begin{aligned}
& \frac{d}{d \Theta}\left\{\frac{1}{\langle k\rangle} \sum_{k} k P(k)\right. \\
& \times\left[I_{k}^{i}(t)=\left(\sigma \lambda k\left(1-\frac{\delta \Omega}{\sigma}\right) \Theta(t) \frac{2 \sum_{i}^{n} \zeta_{i j}}{n^{2}+n}\right)\right. \\
& \times\left((1+\sigma) \lambda k\left(1-\frac{\delta \Omega}{\sigma}\right)\right. \\
& \left.\left.\left.\times \Theta(t) \frac{2 \sum_{i}^{n} \zeta_{i j}}{n^{2}+n}+\sigma\right)^{-1}\right]\right\}\left.\right|_{\Theta=0} \\
& =\frac{\lambda}{\langle k\rangle} \sum_{k} k P(k)\left(1-\frac{\delta \Omega}{\sigma}\right) \frac{2 \sum_{i}^{n} \zeta_{i j}}{n^{2}+n} \geq 1,
\end{aligned}
$$

$\Omega$ is the probability of any given node (individual) which is a neighbor of some specific nodes. Therefore,

$$
\Omega=\frac{k P(k)}{N\langle k\rangle} .
$$

As for BA scale-free network,

$$
P(k)=\frac{2 m^{2}}{k^{3}} .
$$

By substituting (12) and (11) into the lhs of inequality (10), we obtain

$$
\frac{\lambda}{\langle k\rangle}\left[\left\langle k^{2}\right\rangle-\frac{2 m^{2} \delta}{N\langle k\rangle \sigma}\right] \frac{2 \sum_{i}^{n} \zeta_{i j}}{n^{2}+n} \geq 1 .
$$

The value of $\lambda$ satisfies the inequality (13); that is,

$$
\frac{\lambda}{\langle k\rangle}\left[\left\langle k^{2}\right\rangle-\frac{2 m^{2} \delta}{N\langle k\rangle \sigma}\right] \frac{2 \sum_{i}^{n} \zeta_{i j}}{n^{2}+n}=1 .
$$

From the above equation, the critical epidemic threshold $\lambda_{c}$, that is given by

$$
\lambda_{c}=\frac{N\langle k\rangle \sigma}{\left(2 \sum_{i}^{n} \zeta_{i j} /\left(n^{2}+n\right)\right)\left(N\left\langle k^{2}\right\rangle \sigma-2 m^{2} \delta\right)} .
$$

When $\sigma=1$ and $\delta=0, \zeta \rightarrow 1, \lambda_{c}=\langle k\rangle /\left\langle k^{2}\right\rangle$. This is consistent with May's reference.

Here,

$$
\begin{gathered}
\langle k\rangle=\sum_{k} k P(k)=\sum_{k} \frac{2 m^{2}}{k^{2}}=2 m^{2} \sum_{k} \frac{1}{k^{2}} \\
\left\langle k^{2}\right\rangle=\sum_{k} k^{2} P(k)=\sum_{k} \frac{k^{2} 2 m^{2}}{k^{3}}=2 m^{2} \sum_{k} \frac{1}{k} .
\end{gathered}
$$

Substitute (16) into (15)

$$
\begin{aligned}
\lambda_{c} & =\frac{2 N m^{2}\left(\sum_{k}\left(1 / k^{2}\right)\right)^{2} \sigma}{\left[2 N m^{2}\left(\sum_{k}\left(1 / k^{2}\right)\right) \sum_{k}(1 / k) \sigma-\delta\right]\left(2 \sum_{i}^{n} \zeta_{i j} /\left(n^{2}+n\right)\right)} \\
& =\left\{\frac{\sum_{k}(1 / k)}{\sum_{k}\left(1 / k^{2}\right)}-\frac{\delta}{2 N m^{2}\left(\sum_{k}\left(1 / k^{2}\right)\right)^{2} \sigma}\right\} \frac{n^{2}+n}{2 \sum_{i}^{n} \zeta_{i j}} .
\end{aligned}
$$

In the continuous $k$ approximation, calculate the approximation of $\sum_{k}(1 / k)$ and $\sum_{k}\left(1 / k^{2}\right)$. Consider

$$
\begin{aligned}
& \sum_{k} \frac{1}{k} \longrightarrow \int_{m}^{M} \frac{1}{k} d k=\ln \frac{M}{m} \\
& \sum_{k} \frac{1}{k^{2}} \longrightarrow \int_{m}^{M} \frac{1}{k^{2}} d k=\frac{1}{m}-\frac{1}{M}=\frac{M-m}{m M} .
\end{aligned}
$$

Here, $M$ is the maximum degree. By substituting (18) into (17),

$$
\begin{aligned}
\frac{1}{\lambda_{c}} \approx & \left\{\frac{\ln (M / m)}{(M-m) / m M}-\frac{\delta}{2 N m^{2}((M-m) / m M)^{2} \sigma}\right\} \\
& \times \frac{2 \sum_{i}^{n} \zeta_{i j}}{n^{2}+n} \\
= & \left\{\frac{m M \ln (M / m)}{M-m}-\frac{M^{2} \delta}{2 N(M-m)^{2 \sigma}}\right\} \frac{2 \sum_{i}^{n} \zeta_{i j}}{n^{2}+n} .
\end{aligned}
$$

When $M$ is big enough (when $m$ is constant, $M$ increases with $N), M-m \approx M$; thus,

$$
\frac{1}{\lambda_{c}} \approx\left\{m \ln \frac{M}{m}-\frac{M \delta}{2 N \sigma}\right\} \frac{2 \sum_{i}^{n} \zeta_{i j}}{n^{2}+n} .
$$

From (20), we can see the following:

(1) $\lambda_{c}$ increases with $\zeta$ decreasing;

(2) $\lambda_{c}$ increases with $\sigma$; this is reasonable and consistent with expectation; namely, longer lasting immunity raises the threshold infection spreading rate for epidemic outbreak to occur; for simplicity, in this paper, we select $\sigma$ as a fixed value 1 ; 


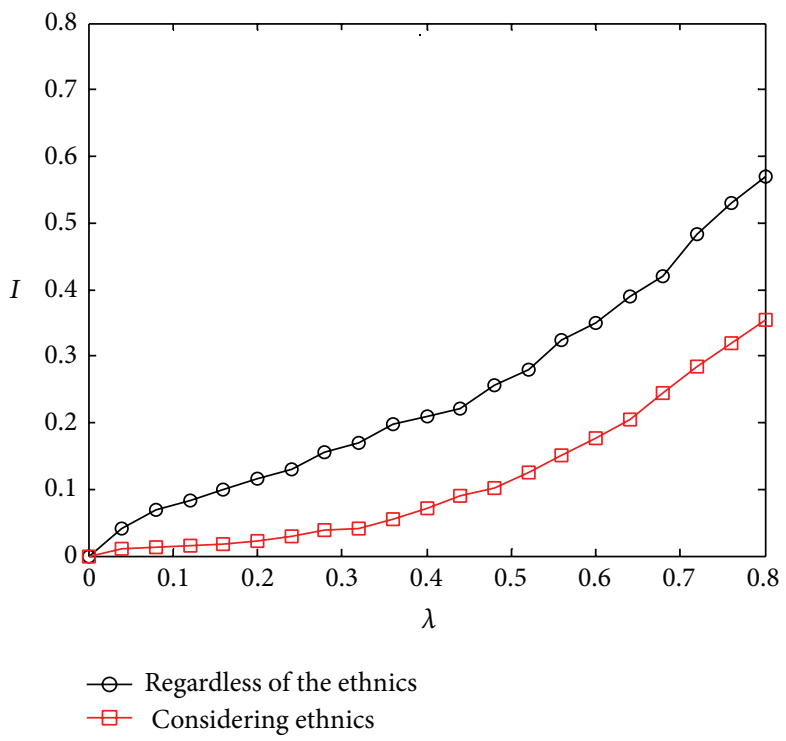

FIGURE 1: Computer simulations of SIRS model on BA scale-free networks $(N=2000,\langle k\rangle=4)$. In the BA scale-free networks, we do not adopt any immunization strategies, $\zeta_{A B}=\zeta_{B C}=\zeta_{A C}=0.7$.

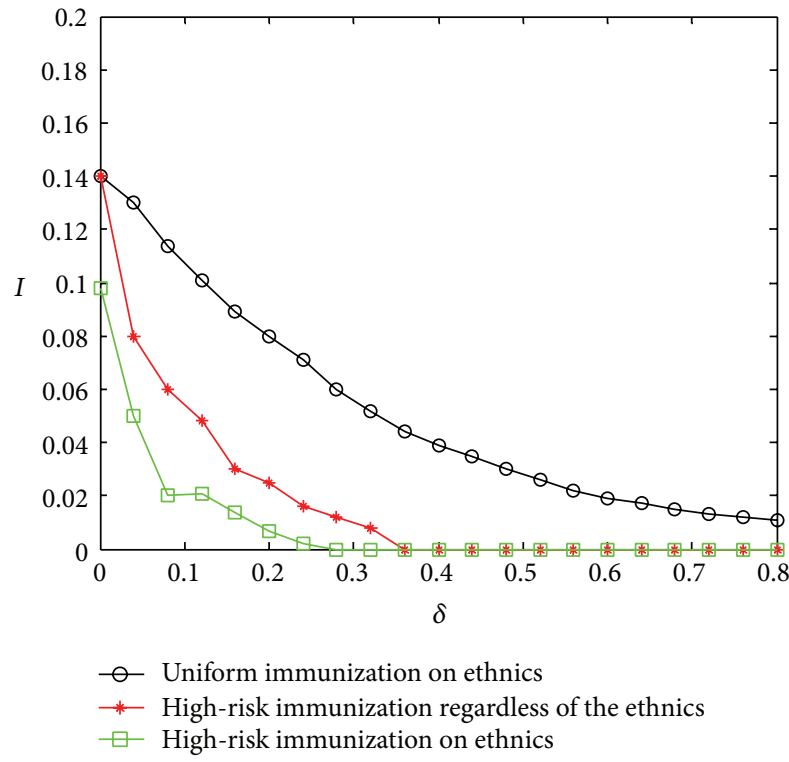

(a) Comparison between uniform immunization and high-risk immunization thinking of ethnics with different probabilities of the immune

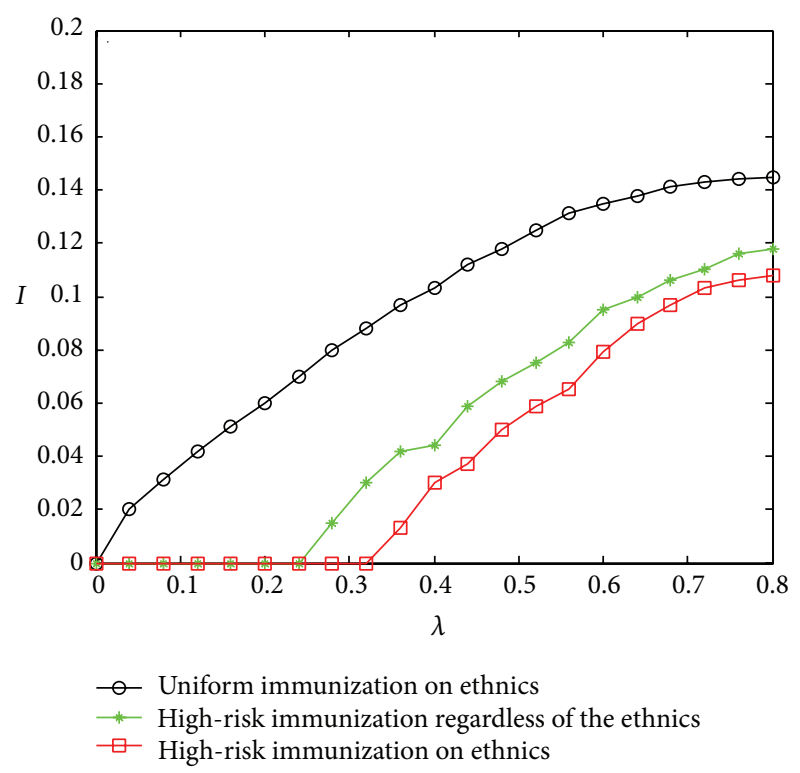

(b) Comparison between uniform immunization and high-risk immunization thinking of ethnics with different transmission probabilities

FIgURE 2: Computer simulations of SIRS model on BA scale-free networks $\left(N=2000,\langle k\rangle=4, \zeta_{A B}=\zeta_{B C}=\zeta_{A C}=0.7\right)$. (a) At a fixed spreading rate $\lambda=0.3$, the changing of infected density $I(\delta)$ as a function of $\delta$. (b) At a fixed vaccinating rate $\delta=0.3$, the changing of infected density $I(\lambda)$ as a function of $\lambda$.

(3) $\lambda_{c}$ increases with $m(\langle k\rangle=2 m)$ decreasing; the simulations can verify this point;

(4) $\lambda_{c}$ decreases with $N$ increasing;

(5) $\lambda_{c}$ increases with $\delta$ increasing; this point is consistent with the simulation.
All the simulations (Figures 1-4) are computed averaging over 30 different starting configurations performed on 20 different realizations of the network. We randomly chose $10 \%$ of nodes as $A$ nation and $20 \%$ of nodes as $B$ nation; the rest of nodes are in $C$ nation.

From Figure 1, we can obviously see that the infection density adopting ethnic distribution strategy runs faster than 


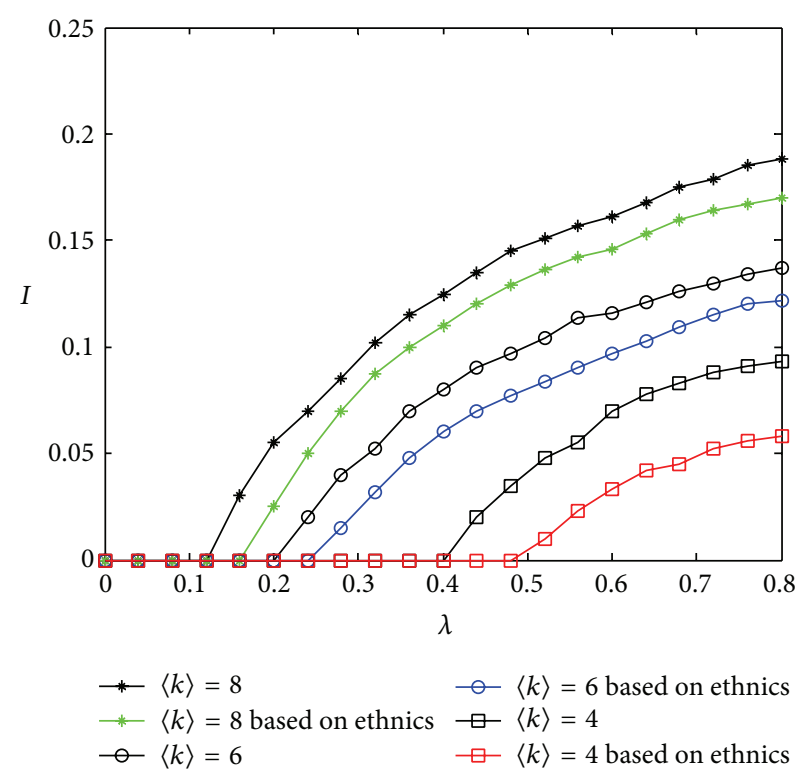

(a) Comparison between high-risk immunization thinking of ethnics and the contrast with different average degrees

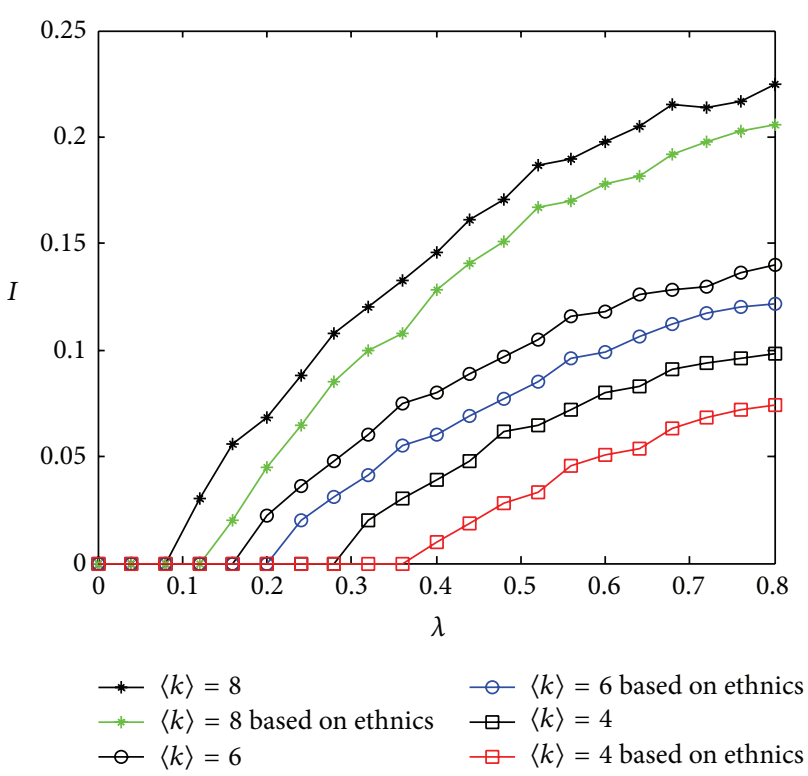

(b) Comparison between high-risk immunization thinking of ethnics and the contrast with different average degrees

FIGURE 3: At a fixed vaccinating rate $\delta=0.3$, the computer simulations of SIRS model with high-risk immunization on BA scale-free networks. $\zeta_{A B}=\zeta_{B C}=\zeta_{A C}=0.7$. (a) When $N=1000$, the variation of infected density $I(\lambda)$ as a function of $\lambda$. (b) When $N=3000$, the changing of infected density $I(\lambda)$ as a function of $\lambda$.

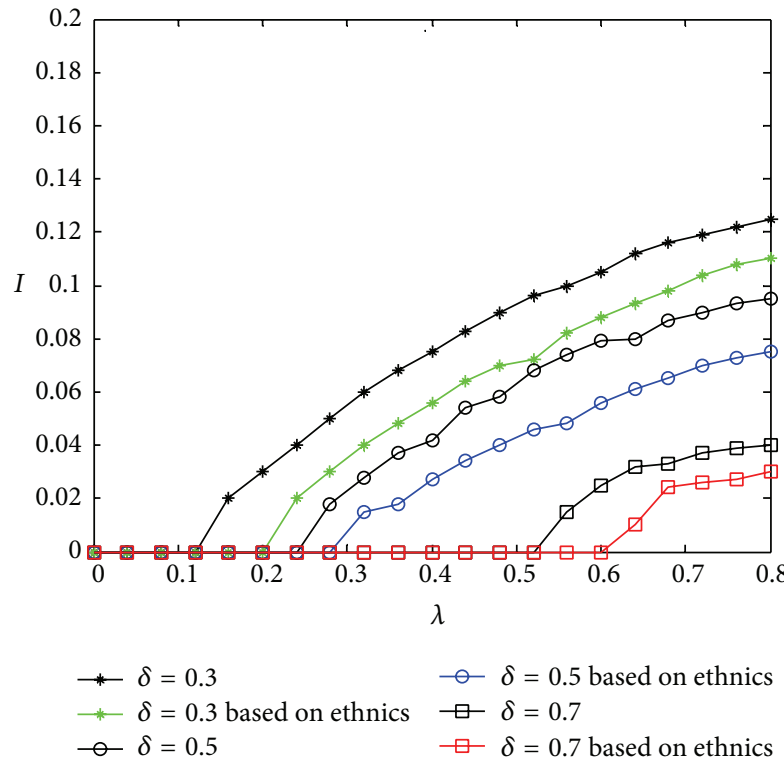

(a) Comparison between high-risk immunization thinking of ethnics and the contrast with different probabilities of the immune

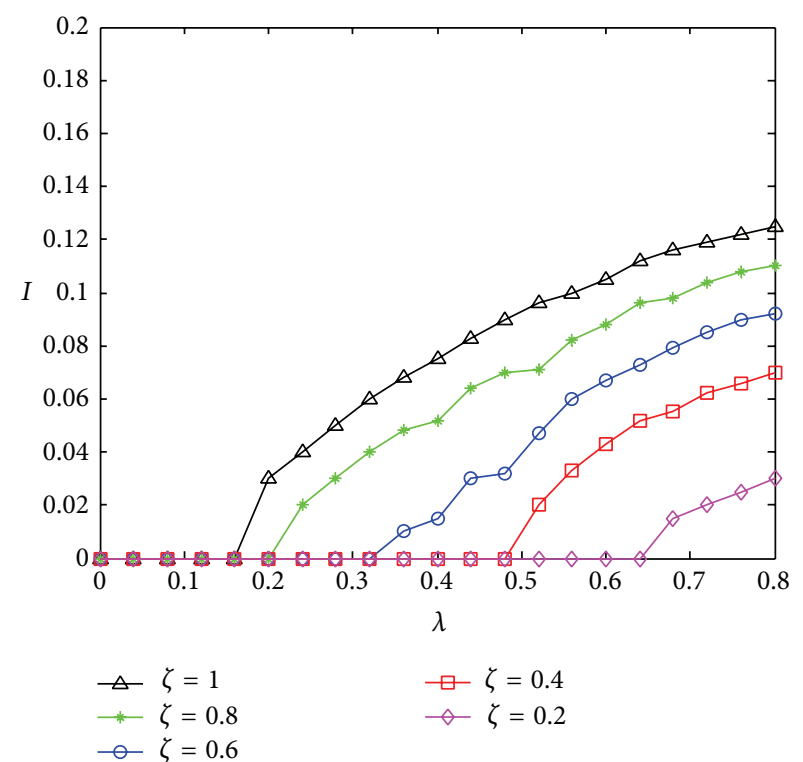

(b) Comparison between high-risk immunization thinking of ethnics and the contrast with different coefficients between ethnics

FIGURE 4: The computer simulations of SIRS model with high-risk immunization on BA scale-free networks $(N=2000,\langle k\rangle=6)$. (a) The changing of infected density $I(\lambda)$ as a function of $\lambda$, with $\delta=0.3, \delta=0.5$, and $\delta=0.7 . \zeta_{A B}=\zeta_{B C}=\zeta_{A C}=0.7$. (b) The changing of infected density $I(\lambda)$ as a function of $\lambda$, with $\zeta=0.2, \zeta=0.4, \zeta=0.6, \zeta=0.8$, and $\zeta=1\left(\zeta_{A B}=\zeta_{B C}=\zeta_{A C}=\zeta\right)$. $\delta=0.3$.

another situation. This is consistent with the true life. To get close to actual conditions in the multiethnic regions, we should use consider the race property of nodes.

From Figure 2(a), we can see that the density of infected nodes is decreased with vaccinating rate $\delta$ increasing; besides when we adopt ethnic distribution strategy, it has an immune effect by itself. Figure 2(b) shows that the density of infected node is increased with spreading rate $\lambda$ increasing; this is consistent with the above analysis in BA scale-free networks. And Figure 2 also shows that the high-risk immunization 
in the multiethnic regions is effective, but the uniform immunization is not.

From Figure 3, we can see the $\lambda_{c}$ increases with $m(\langle k\rangle=$ $2 m)$ decreasing. This is verified by the above analysis. It also shows that $\lambda_{c}$ decreases with $N$ increasing, as found in the above analysis.

Figure 4 indicates that $\lambda_{c}$ increases with vaccinating rate $\delta$ increasing, and this is also consistent with the above analysis. This indicates that as long as the vaccinating rate is big enough, the epidemic would not be prevalent in population. (If the value of $\lambda$ is above the threshold, $\lambda \geq \lambda_{c}$, the infection spreads and becomes persistent. Below threshold, the infection dies out exponentially fast.) On the other hand, it indicates that when the $\zeta$ is big enough, it has a weaker impact on high-risk immunization. Especially, if $\zeta=1$, there is no impact on high-risk immunization.

\section{Conclusions}

Immunization is an interesting and challenging task, which aims at less cost and better effect. We analyzed the effectiveness of high-risk immunization from a theoretical point of view for multiethnic regions and established models on scalefree networks. Considering the factors of politics, nations, economies, and so forth, neither "acquaintance immunization" nor "targeted immunization schemes" can be used in practice. Moreover, targeted immunization needs the whole information of the network. This is often very hard to get. Uniform immunization is a simple strategy, but it is not effective. And worst of all, it requires vaccination of almost the entire population, which is costly and administratively unfeasible. High-risk immunization for multiethnic areas is a dynamic scheme and, at each time step, requires vaccination of only the part of neighbors of infected individuals. The cost is relatively small. Besides, it is very close to social reality. At the same time, it is similar to acquaintance immunization. So, high-risk immunization not only has its own advantages but also its own high-risk immunization's merit.

\section{Conflict of Interests}

The authors declare that there is no conflict of interests regarding the publication of this paper.

\section{Acknowledgments}

This research is supported by the National Natural Science Foundation of China (no. 61263019), the Fundamental Research Funds for the Gansu Universities (no. 1114ZTC144), the Natural Science Foundation of Gansu province (no. 1112RJZA029), and the Doctoral Foundation of LUT.

\section{References}

[1] H.-X. Yang, W.-X. Wang, Y.-C. Lai, Y.-B. Xie, and B.-H. Wang, "Control of epidemic spreading on complex networks by local traffic dynamics," Physical Review E: Statistical, Nonlinear, and Soft Matter Physics, vol. 84, no. 4, Article ID 045101, 2011.
[2] J. S. Haukoos, E. Hopkins, A. Hull et al., "HIV testing in emergency departments in the United States: a national survey," Annals of Emergency Medicine, vol. 58, no. 1, pp. S10-S16, 2011.

[3] X. Chu, Z. Zhang, J. Guan, and S. Zhou, "Epidemic spreading with nonlinear infectivity in weighted scale-free networks," Physica A: Statistical Mechanics and its Applications, vol. 390, no. 3, pp. 471-481, 2011.

[4] S. Merler and M. Ajelli, "Human mobility and population heterogeneity in the spread of an epidemic," Procedia Computer Science, vol. 1, no. 1, pp. 2237-2244, 2010.

[5] K. Li, M. Small, H. Zhang, and X. Fu, "Epidemic outbreaks on networks with effective contacts," Nonlinear Analysis: Real World Applications, vol. 11, no. 2, pp. 1017-1025, 2010.

[6] G. González-Parra, L. Acedo, R.-J. Villanueva Micó, and A. J. Arenas, "Modeling the social obesity epidemic with stochastic networks," Physica A: Statistical Mechanics and its Applications, vol. 389, no. 17, pp. 3692-3701, 2010.

[7] R. M. May and A. L. Lloyd, "Infection dynamics on scale-free networks," Physical Review E: Statistical, Nonlinear, and Soft Matter Physics, vol. 64, no. 6, Article ID 066112, 2001.

[8] R. Pastor-Satorras and A. Vespignani, "Epidemic spreading in scale-free networks," Physical Review Letters, vol. 86, no. 14, pp. 3200-3203, 2001.

[9] M. Barthélemy, A. Barrat, R. Pastor-Satorras, and A. Vespignani, "Velocity and hierarchical spread of epidemic outbreaks in scale-free networks," Physical Review Letters, vol. 92, no. 17, Article ID 178701, 2004.

[10] J.-P. Zhang and Z. Jin, "The analysis of an epidemic model on networks," Applied Mathematics and Computation, vol. 217, no. 17, pp. 7053-7064, 2011.

[11] K. Gao and D. Y. Hua, "Effects of immunity on global oscillations in epidemic spreading in small-world networks," Physics Procedia, vol. 3, no. 5, pp. 1801-1809, 2010.

[12] Z.-P. Li and G.-L. Shao, "Halting infectious disease spread in social network," in Proceedings of the International Workshop on Chaos-Fractals Theories and Applications (IWCFTA '09), pp. 305-308, November 2009.

[13] K. T. D. Eames, J. M. Read, and W. J. Edmunds, "Epidemic prediction and control in weighted networks," Epidemics, vol. 1, no. 1, pp. 70-76, 2009.

[14] J. H. Sorensen, S. Alexandersen, P. Astrup et al., "The VetMet veterinary decision support system for airborne animal diseases," in Air Pollution Modeling and Its Application XIX, NATO Science for Peace and Security Series, pp. 199-207, 2008.

[15] M. Pautasso and M. J. Jeger, "Epidemic threshold and network structure: the interplay of probability of transmission and of persistence in small-size directed networks," Ecological Complexity, vol. 5, no. 1, pp. 1-8, 2008.

[16] F. Nian and X. Wang, "Efficient immunization strategies on complex networks," Journal of Theoretical Biology, vol. 264, no. 1, pp. 77-83, 2010.

[17] D. S. Callaway, M. E. J. Newman, S. H. Strogatz, and D. J. Watts, "Network robustness and fragility: percolation on random graphs," Physical Review Letters, vol. 85, no. 25, pp. 5468-5471, 2000.

[18] R. Pastor-Satorras and A. Vespignani, "Immunization of complex networks," Physical Review E: Statistical, Nonlinear, and Soft Matter Physics, vol. 65, no. 3, Article ID 036104, pp. 1-8, 2002. 


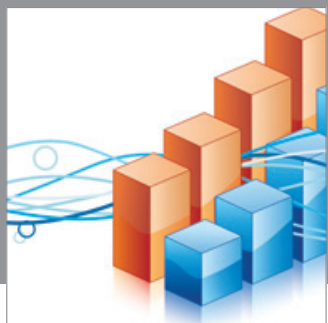

Advances in

Operations Research

mansans

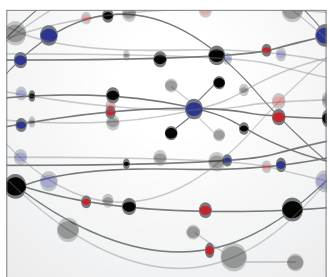

The Scientific World Journal
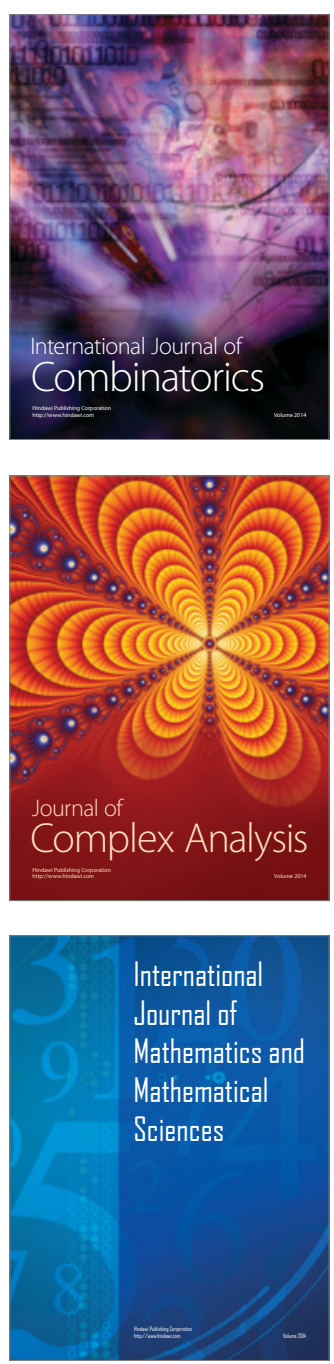
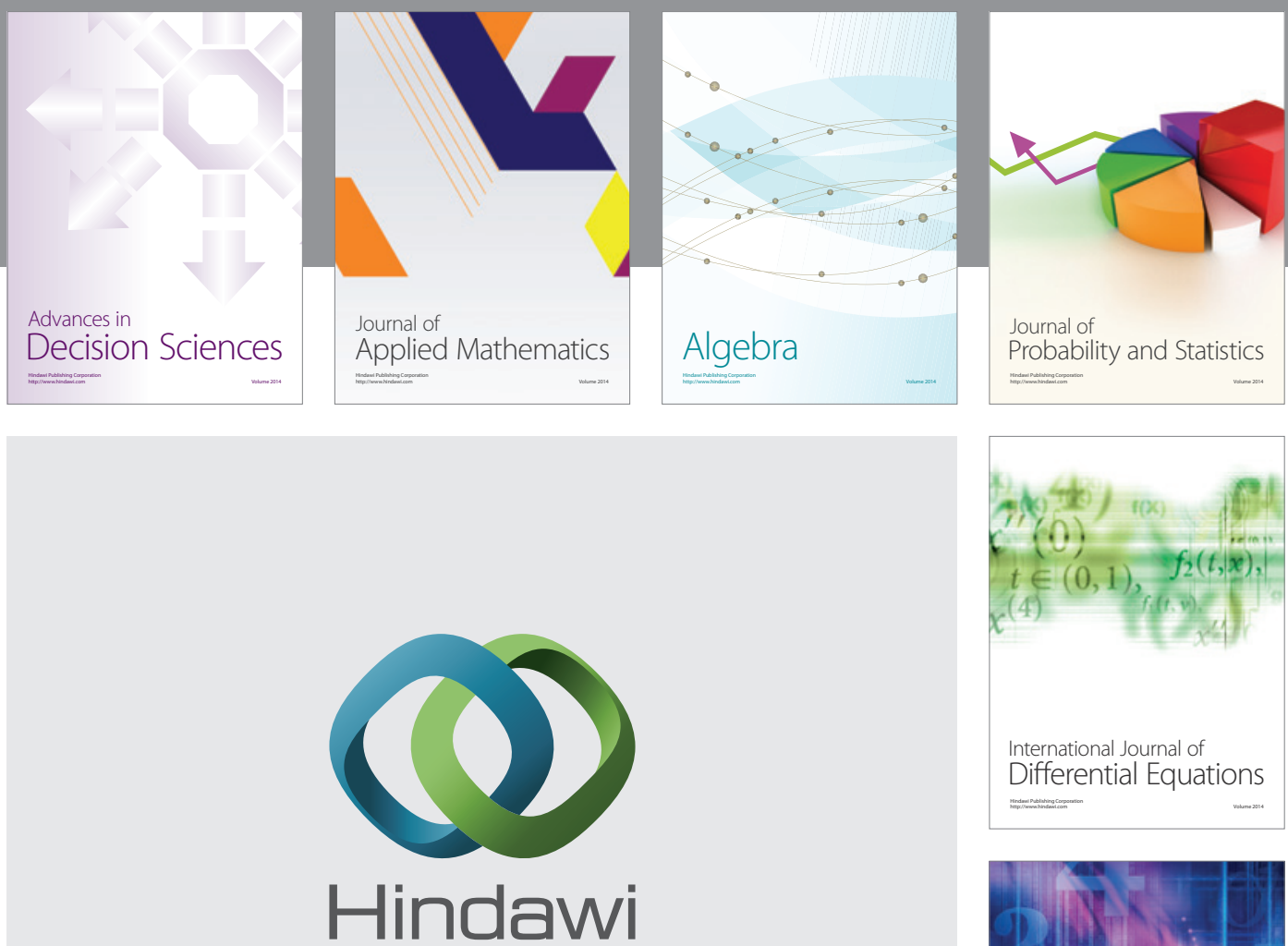

Submit your manuscripts at http://www.hindawi.com
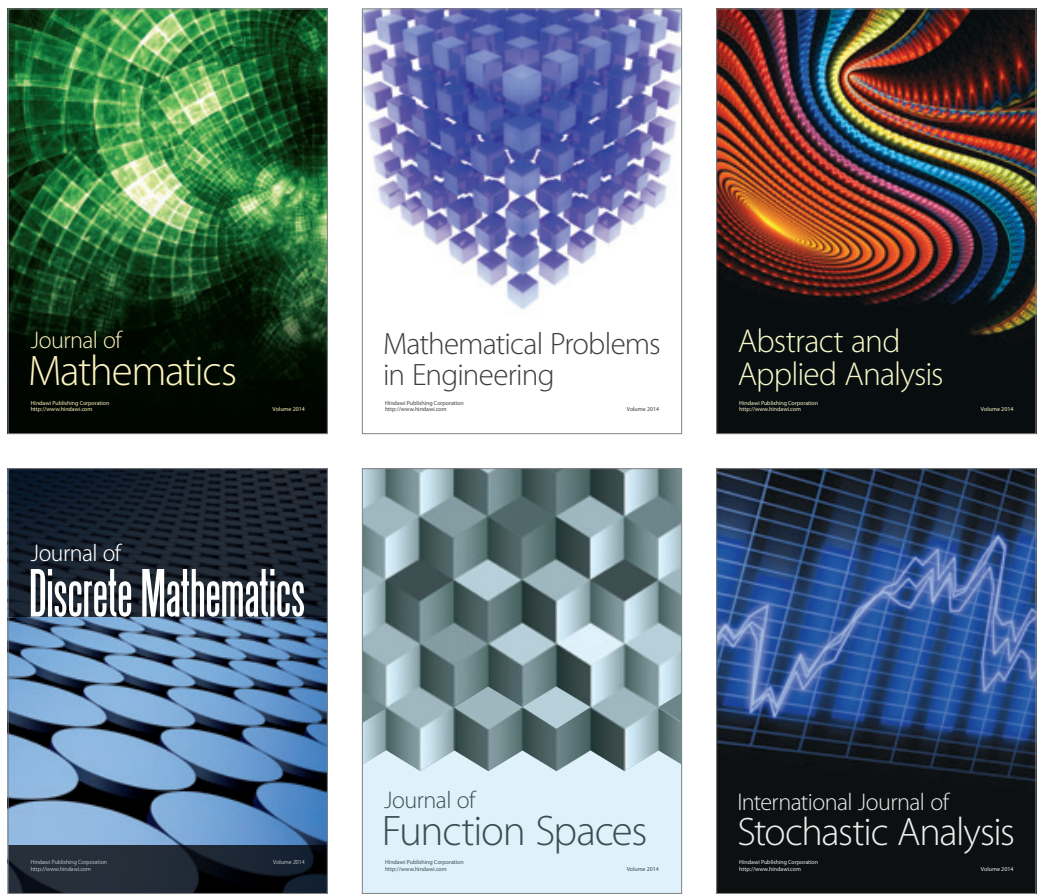

Journal of

Function Spaces

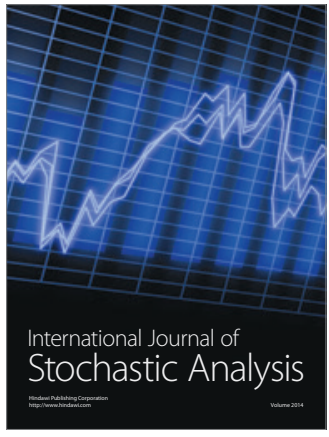

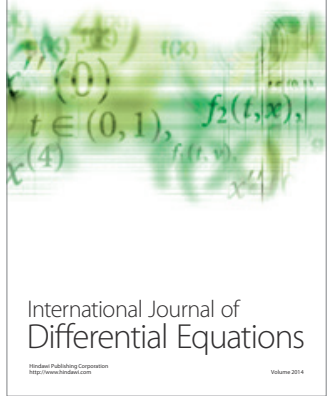
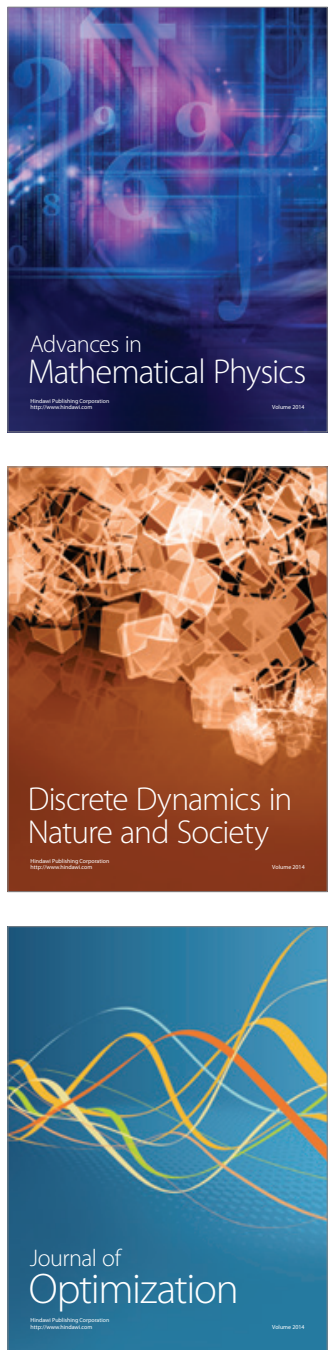